\title{
Long-Term Outcome of Infection Control for Methicillin-Resistant Staphylococcus aureus and Kaizen Approach with Problem-Solving
}

\author{
Takanori Ayabe1,2,3*, Masaki Tomita1, Ichiro Takajo4, Yuki Nakayama4, Ryo Maeda1, \\ Manabu Okumura' ${ }^{2}$, Kunihide Nakamura ${ }^{2,5}$

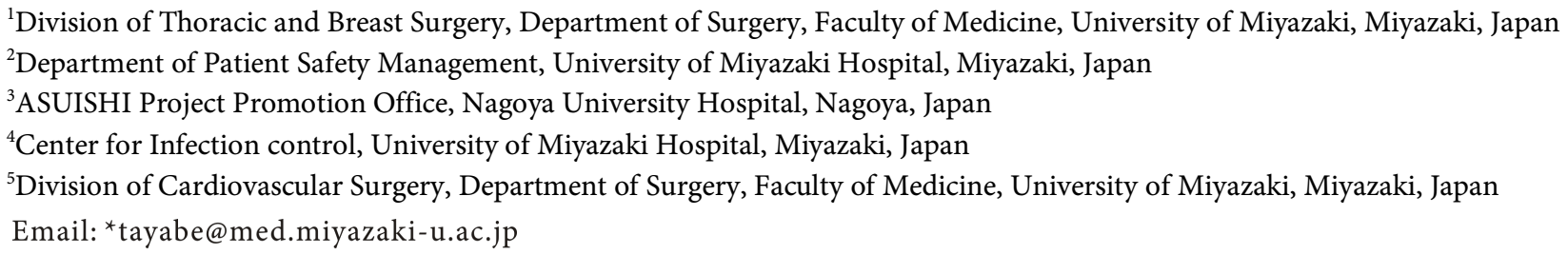

How to cite this paper: Ayabe, T., Tomita, M., Takajo, I., Nakayama, Y., Maeda, R., Okumura, M. and Nakamura, K. (2020) Long-Term Outcome of Infection Control for Methicillin-Resistant Staphylococcus aureus and Kaizen Approach with Problem-Solving. Open Journal of Safety Science and Technology, 10, 1-23.

https://doi.org/10.4236/ojsst.2020.101001

Received: January 5, 2020

Accepted: February 25, 2020

Published: February 28, 2020

Copyright (C) 2020 by author(s) and Scientific Research Publishing Inc. This work is licensed under the Creative Commons Attribution International License (CC BY 4.0).

http://creativecommons.org/licenses/by/4.0/ (c) (i) Open Access

\begin{abstract}
Background: Methicillin-resistant Staphylococcus aureus (MRSA) results in longer hospitalization, increased expenses, and poorer patient prognosis. The aim of this study is 1) to investigate the short-term outcome of MRSA outbreak and the prevention in our surgical ward, and 2) to evaluate the long-term outcome of a 7-year experience of infection control, and 3) to report the effectiveness of intervention of quality improvement by industry problem-solving method for the eradication of a new occurrence of MRSA patients. Methods: Between April 2009 and October 2019, we retrospectively studied the improvement activity for infection control and preventative measures in our surgical ward. The daily alcohol use for hand hygiene was measured ( $\mathrm{ml} /$ patient/day) and the monthly number of occurrences of new MRAS patients was investigated. We used the industry problem-solving method as the Kaizen of infection control for the eradication of a new Methicillin-resistant Staphylococcus aureus occurrence in the surgical ward. Results: There was an inverse correlation between the increased amount of alcohol use for hand hygiene and the decreased number of new MRSA patients. In the long-term outcome, the amount of alcohol use has gradually decreased and the MRSA patients have been occasionally observed; these facts should display the difficulty to maintain a strict infection control. By the use of the problem-solving method, there continued for 7 months no new MRSA patients between June 2018 and December 2018. However, once the amount of alcohol use for hand hygiene decreased below $40 \mathrm{ml} /$ patient/day, three new MRSA patients were detected. It displayed an inverse correlation between the
\end{abstract}


decreased amount of alcohol use for hand hygiene and the increased number of new MRSA patients. Conclusion: The daily monitoring and measuring of the amount of alcohol use for hand hygiene and to know the current number of new occurrence of MRSA patients will become a meaningful tool. By performing the Kaizen with a problem-solving method, it will contribute to the multi-professional team to visualize the process of quality improvement for infection control.

\section{Keywords}

MRSA, Alcohol, Hand Hygiene, Infection Control, Kaizen, Problem-Solving

\section{Introduction}

Hand hygiene is widely recognized and promoted as a simple but effective practice to counter incidences of health care-acquired infections. However, worldwide compliance is less than optimal [1] [2]. According to reports from the Center for Disease Control and Prevention, healthcare workers do not clean their hands as often as they should [3]. Poor hand hygiene compliance among healthcare workers contributes to the spread of devastating health care-associated infection, and consequently, prolonged patient's hospital stays, disability, increased resistance to antibiotics, increased mortality and high health care costs [1].

Methicillin-resistant Staphylococcus aureus (MRSA) results in longer hospitalizations, increased expenses, and poorer patient prognosis. MRSA has been rapidly increasing worldwide over the past several decades [4]. MRSA transmission is common in hospitals, where it is spread from patient to patient on health care worker's hands by contaminated environments, or directly from patient to patient [5]. Additionally, hospitalized patients are vulnerable to MRSA infections because they often have indwelling devices, are immunosuppressed, or have had surgical procedures [5]. Treatment options are limited for patients with MRSA infections.

Poor hand hygiene is the main source of MRSA transmission within a hospital [6]. However, after applying alcohol gel, $99 \%$ of the transient organisms, including MRSA, are eradicated [7]. In an attempt to reduce the incidence of patients with MRSA, hand-hygiene awareness has become more prominent worldwide [8] [9]. Research has also identified numerous other potential sources of MRSA within hospitals and studies have proposed that healthcare workers may act as a source for MRSA transmission [10]. All persons entering a surgical ward may therefore act as an exogenous source of MRSA to susceptible patients, especially if hand-hygiene policies are not adhered to [11]. It is therefore imperative for all persons entering a surgical ward to apply alcohol gel as a means of reducing nosocomial MRSA [11].

Our surgical ward has many high-risk patients with severe complications be- 
fore and after surgery, i.e., cardiovascular surgery using artificial valves and vascular grafts, and also thoracic and digestive surgeries. In May 2011, we had 10 MRSA patients in 49 beds and experienced an outbreak. We postponed all scheduled operations and separated the MRSA patients. The occurrence of the outbreak of MRSA has a big impact on society. By the use of strict infection standard preventative and contact preventive measures, it took 3 months to reduce the outbreak. We then continued the strict infection control measures for seven years. However, the novel experience of the outbreak was forgotten, and a thorough hand hygiene and manual compliance of infection control were lost. The new infection of MRSA has not been erased, thus we found it difficult to maintain a strict infection control with a sense of tension and it became a challenge.

The aim of the present study is 1) to summarize the retrospective analysis regarding the short-term outcome of our past MRSA outbreak and the prevention, and 2) to evaluate the long-term outcome of seven years of infection control after the prevention of the outbreak, and 3) to report the outcome of the intervention of the Kaizen approach with an industry problem solving method for eradication of a new occurrence of MRSA. When newly setting the goals of prevention of a new occurrence of the positive detection of MRSA, we implemented an industry total quality management and problem-solving method for infection control.

\section{Methods}

\subsection{Study Design}

A retrospective analysis was performed on our past experience of the MRSA outbreak and prevention in 2011. For 9 years between April 2009 and April 2018, the long-term outcome of infection control was investigated. For 22 months between January 2018 and October 2019, the industry problem solving approach was implemented for the extermination of a new Methicillin-resistant Staphylococcus aureus occurrence in the surgical ward. The outcome of the quality improvement was evaluated. This study was approved by the University of Miyazaki Hospital Clinical Research Ethics Board (No. 2017-166, C-0033) in March 2018.

\subsection{Situation of the Surgical Ward before the Outbreak (before May 2011)}

Our surgical ward has 49 patient beds where were used by the three surgical teams consisting of cardiovascular, thoracic, and digestive surgeries. Most all the hospitalized patients consisted of elderly patients with severe complications. Surgeons performed the major surgery on the average of 10 to 15 operations per week, the majority of which were classified as high risk with the systemic administration including emergency surgery. Three surgical teams as the attending physicians managed the presurgical treatment of these patients, making the de- 
cision of surgical procedures, and the post-operative care. In principle, the doctor in charge of the day of the week performs the sanitation disinfection in cooperation with the nurse in charge of the ward, except for patients with severe infections that require special cleaning and the method of attachment.

\subsection{Background of the MRSA Outbreak in the Surgical Ward (in May 2011)}

In May 2011, four MRSA patients had been hospitalized in our surgical ward. In addition, the other four MRSA patients had been newly detected. As a result, we had eight MRSA patients in the same surgical ward, thus the so-called status of MRSA outbreak occurred. On May 30, 2011, the nosocomial center of infection control (CIC) started the intervention of infection control to our surgical ward. We had two other new MRSA patients. Finally, we faced the situation of a total of ten MRSA patients in the same ward (Table 1 and Table 2).

Table 1. Background of the MRSA patients.

\begin{tabular}{|c|c|c|c|c|c|c|c|c|}
\hline Cases & Surgical Teams & $\begin{array}{c}\text { New } \\
\text { occurrence }\end{array}$ & $\begin{array}{l}\text { Similarity of } \\
\text { drug } \\
\text { susceptibility }\end{array}$ & $\begin{array}{c}\text { Same } \\
\text { room at } \\
\text { the same } \\
\text { period }\end{array}$ & $\begin{array}{l}\text { Nursing } \\
\text { necessity }\end{array}$ & $\begin{array}{c}\text { Age, } \\
\text { gender }\end{array}$ & Diagnosis & $\begin{array}{l}\text { Surgery and medical } \\
\text { treatment }\end{array}$ \\
\hline 1 & Caridovasucular & & O & A room & + & $64 \mathrm{M}$ & Angina, heart failure & $\begin{array}{l}\text { Coronary artery bypass ( } 3 \\
\text { vessels), ventricular drainage }\end{array}$ \\
\hline 3 & Caridovasucular & $\bigcirc$ & & A room & + & $84 \mathrm{M}$ & $\begin{array}{l}\text { Arteriosclerosis } \\
\text { obliterans, } \\
\text { graft-infection of rt. } \\
\text { femoro-popliteal } \\
\text { bypass }\end{array}$ & $\begin{array}{l}\text { Removal of infected graft, } \\
\text { arterial angioplasty }\end{array}$ \\
\hline 4 & Caridovasucular & & $\bigcirc$ & B room & + & $83 \mathrm{~F}$ & $\begin{array}{l}\text { Abdominal aortic aneu- } \\
\text { rysm }\end{array}$ & $\begin{array}{l}\text { Stent interpolation, rupture, } \\
\text { replacement of graft, } \\
\text { hemostatic surgery for } \\
\text { the ruptured proximal } \\
\text { anastomosis, }\end{array}$ \\
\hline 5 & Digestive & $\bigcirc$ & O & B room & + & $73 \mathrm{~F}$ & Gall bladder cancer & $\begin{array}{l}\text { Percutaneous transhepatic } \\
\text { bile duct drainage }\end{array}$ \\
\hline 6 & Digestive & $\bigcirc$ & $\bigcirc$ & B room & + & $86 \mathrm{M}$ & Gastric cancer & $\begin{array}{l}\text { Anastomsis of gastric and } \\
\text { jejunum }\end{array}$ \\
\hline 8 & Thoracic & $\bigcirc$ & $\bigcirc$ & - & - & $79 \mathrm{M}$ & Lung cancer & Right lower lobectomy \\
\hline 9 & Thoracic & $\bigcirc$ & & - & - & $66 \mathrm{M}$ & Lung cancer & Right lower lobectomy \\
\hline 10 & Thoracic & & & - & - & $65 \mathrm{M}$ & Lung cancer & $\begin{array}{l}\text { Right lower lobectomy, } \\
\text { partial resection of S2 }\end{array}$ \\
\hline
\end{tabular}


Table 2. Short-term outcome of the MRSA patients.

\begin{tabular}{|c|c|c|c|c|}
\hline Cases & Complications & Detected site of MRSA & Clinical course of MRSA & Outcomes \\
\hline 1 & $\begin{array}{l}\text { Postoperative infarction and cerebellum } \\
\text { water head disese with mediastinal } \\
\text { sinusitis, (filling of large pectoral } \\
\text { muscle flap) }\end{array}$ & $\begin{array}{l}\text { Blood, cerebrospinal fluid, } \\
\text { phlegm, clogged pus }\end{array}$ & infection continued, settled & recovery, transference \\
\hline 2 & $\begin{array}{l}\text { Acute renal failure with mediastinal } \\
\text { sinusitis, (filling of abdominal straight } \\
\text { muscle flap) }\end{array}$ & $\begin{array}{l}\text { Blood, pleural effusion, } \\
\text { phlegm, open pus }\end{array}$ & infection continued & $\begin{array}{l}\text { death from renal } \\
\text { failure, discharged }\end{array}$ \\
\hline 3 & Infection of surgical wound site & Open pus & $\begin{array}{l}\text { infection continued, healed, } \\
\text { disappeared }\end{array}$ & recovery, dischage \\
\hline 4 & Postoperative hemorrhage & Blood, Open pus & infection continued, settled & transference \\
\hline 5 & $(-)$ & Open pus & $\begin{array}{l}\text { infection continued, healed, } \\
\text { disappeared }\end{array}$ & recovery, transference \\
\hline 6 & $\begin{array}{l}\text { Infection of central venous } \\
\text { catheterization with fever }\end{array}$ & Blood & $\begin{array}{l}\text { infection continued, healed, } \\
\text { disappeared }\end{array}$ & recovery, transference \\
\hline 7 & Infection of surgical wound site & Open pus, phlegm & infection continued, settled & recovery, transference \\
\hline 8 & Postoperative pneumonia & Phlegm & $\begin{array}{l}\text { infection continued, healed, } \\
\text { disappeared }\end{array}$ & recovery, discharge \\
\hline 9 & Postoperative acute empyema & Open pus & $\begin{array}{l}\text { infection continued, healed, } \\
\text { disappeared }\end{array}$ & recovery, discharge \\
\hline 10 & Postoperative acute empyema & Open pus & $\begin{array}{l}\text { infection continued, healed, } \\
\text { disappeared }\end{array}$ & recovery, discharge \\
\hline
\end{tabular}

\subsection{Responding to the Outbreak (in 2011)}

\subsubsection{Intervention of Nosocomial Center for Infection Control (CIC)}

On May 30, a multi-professional conference was held with three surgical teams, ward doctor, nurse head, deputy nurse head, infection control doctor and nurse, and others. They agreed upon enforcing hand hygiene in the surgical ward, isolation of MRSA-positive patients in private rooms, cohort survey, use of protective equipment such as gloves, and manual compliance. We thoroughly discussed the correspondence for MRSA-positive patients. On June 3, new two MRSA patients were confirmed after the intervention on May 30. We were offered to consider stopping new surgery and new hospitalization for our surgical ward. At the same time, the MRSA outbreak status was reported to the director of the hospital. On June 6, the members of the nosocomial center for infection control (CIC) participated in a conference at our surgical department. Infection preventive measures and agreement on hospitalized patients in the surgical ward were provided.

\subsubsection{Action of Multi-Professional Staff in Surgical Ward}

On May 30, invasive open-heart surgery and the scheduled regular surgery were 
decided to be stopped for the time being (except for emergency surgery). On June 3, all scheduled periodic surgery from June 6 had been suspended (except for urgently requested surgery) due to the additional occurrence of 2 new MRSA patients. On June 7, the measurement of the accurate actual use of hand sanitizer (alcohol consumption) for one patient per day was started. On June 8, we reviewed the infection standard preventive and contact preventive measures and formulated an agreement. On June 13, the cardiovascular surgical team was allowed access to the surgical patients from the internal medicine ward and perform post-operative management in the intensive care unit (ICU), the ICU exit was allowed to exit to the pediatric ward and cardiovascular internal medicine ward with the policy that does not pass through the our hospital ward. The digestive and thoracic surgical teams continued operations for the next 2 weeks. The thoracic surgical team transferred the hospital-waiting patients to another facility, and we asked for surgery. On June 20, the cardiovascular and thoracic surgicalteams resumed operation. The digestive surgicalteam refused to resume surgery. We reviewed the infection standard preventive and determined preventive measures. On June 27, the digestive surgicalteam resumed operation.

\subsection{Infection Prevention and Control for the Inpatients during the MRSA Outbreak}

The nosocomial center for infection control (CIC) classified inpatients as 4 types, that is, severely ill MRSA patients, MRSA-detected patients, non-MRSA general patients, and new hospitalized patients. The CIC instructed us to take appropriate infection measures corresponding to each type and we performed the 4 types of infection measures according to the newly created handling standards.

\subsection{Manual about the Dressing-Exchange of Surgical Site during the Outbreak}

We created a new manual regarding the dressing-exchange at the surgical site during the outbreak, which included standard precautions and infection prevention measures for the MRSA outbreak. All of the multi-professional staff carried the pocket manual describing these contents as a printed version. All the staff agreed 1) a priority on the dressing-exchange of the surgical site, 2) roles of clean and preparatory assistants, 3) standard precautions for wound dressing-exchange, and 4) procedure of dressing-exchange.

\subsubsection{The Priority for the Dressing-Exchange at a Surgical Site}

1) the patients without a bacterial infected wound and the patient without an open surgical site. Do not put the dressing-exchange cart in the patient's room, 2) the patients with an open surgical site and the patient who needs a wound washing, 3) patients with a bacterial infection, and 4) patients with a positive bacterial infection from an open surgical site. 


\subsubsection{Roles of Clean and Preparatory Assistants}

The role of the clean assistant: Do not remove a patient's gauze dressing. Do not put on tape. Do not touch the patient.

The role of the dressing-exchange assistant: Fix gauze. Attach and detach the patient's clothes. Assist with the patient's position during wound-washing. After dressing-exchange of the wound site is done, the assistant must move to the next patient's bed and prepare it. Depending on the progress of the dressing-exchange round, separately moves the filth cart.

\subsubsection{Standard Precautions for Dressing-Exchange}

1) At the time of dressing-exchange of a patient with an open wound in the order of "first, wear gloves", "second, a mask" and "third, an apron". 2) At the time of cleaning a patient with an open wound in the order of "first, wear gloves", "second, a mask", "third, a gown", "fourth, a cap" and "fifth, goggles". 3) At the time of dressing-exchange of a patient with a bacterial infection in the order of "first, wear gloves", "second, a mask" and "third, an apron". Caution: if inclose contact with a patient or patient who has a sore or cough, wear a gown. 4) At the time of usual dressing-exchange without an infected wound in the order of "first, wear gloves" and "second, a mask". Caution: when removing drain tubes, wear an apron.

\subsubsection{Procedure of Dressing-Exchange}

At least 1 doctor and 2 nurses (clean assistant, person in charge of dressing-exchange) are involved in the dressing-exchange process. On weekdays, one doctor is present to observe the entire dressing-exchange process.

Preparation before the dressing-exchange: 1) Use a quick-drying hand sanitizer before entering the patient room. 2) The person in charge of the dressing-exchange opens the patient's clothes, then puts on a blanket. Caution: If you know to wear a gown or apron at the time of dressing-exchange, in advance, prepare a large plastic bag. 3) Use quick-drying hand sanitizer when leaving the patient room (in the case of a 4-patient room, hand disinfection should be thorough for each patient).

Start of dressing-exchange: 1) Use quick-drying hand sanitizer before entering patient room. 2) The clean assistant opens the curtain so that the dressing-exchange cart can enter. Gloves are then worn. For the patients with a cleaning wound, wear protective gear at the entrance of the room. In the case of infected patients or patients who have been totally washed, if the required items are determined, prepare the goods in the tray. Do not put the dressing-exchange cart in the patient room. 3) Discard the removed gauze directly into a plastic bag. When using forceps and scissors, the pus tray is used. 4) At the end of the dressing-exchange, remove gloves and use a quick-drying hand sanitizer, then move on to the next patient. Dispose of protective equipment, such as gowns and aprons, in prepared trash cans or plastic bags. After the end of the dressing-exchange, close and set the plastic bag and discard it. 5) Put the waste tray in 
a plastic bag on the dirt cart and take it out for cleaning.

\subsection{Data Collection and MRSA Surveillance}

Retrospectively, clinical data were collected before and after the MRSA outbreak when the period was between April 2009 and October 2019. Before the outbreak, we did not measure the everyday amount of alcohol use in our surgical ward. From the occurrence of the outbreak, we reviewed the manual of infection control and we started the everyday measuring of the amount of alcohol use. After checking the daily decrease in the alcohol amount in the disinfection alcohol bottle set in front of each patient room and the medical staff s own hand hygiene bottle, the total consumed amount of alcohol had been averaged ( $\mathrm{ml} /$ patient/day), the value of which was displayed on the surgical ward calendar. Every morning, the medical staff will easily know the number of MRSA patients and the status of the prior day's consumption amount of alcohol as a result of the monitoring. If the amount of alcohol use had decreased compared to that of the prior day, we called the medical staff to increase the level of the hand hygiene. We daily recorded the amount of alcohol use and monitored the number of hospitalized patients and a new MRSA patient and the patient's location in the surgical ward for more than 8 years. We have continued to record the everyday work after the outbreak. The system of the daily measured amount of alcohol use for hand hygiene and the hospitalized patient's number play a function of everyday work and act as an everyday indicator of infection control. The monthly average amount of alcohol use was calculated. We have continued to monitor the trends in the occurrence number of new MRSA patients in the surgical ward and everyday average amount of the alcohol use. In the case of a lower level of alcohol use and newly increased occurrence of MRSA patients, we urged an alert of risk of occurrence of the outbreak to the medical staff in the surgical ward.

\subsection{Statistical Analysis}

A statistical technique was used that involved the seven techniques for qualitative analysis, which are collectively called the seven tools for quality control (QC seven tools). To analyze the frequency of appearance of the incident levels of new MRSA patients is part of the quality. The data in this study were analyzed by the breakdown of the MRSA outbreak patients, transition of the number of MRSA patients and the monthly amount of alcohol use. Changes in the variables were analyzed by comparison of before and after the intervention for the MRSA outbreak and before and after the current intervention of the Kaizen approach along with the industry problem-solving method.

\subsection{Intervention of Quality Improvement Using Industry Problem-Solving Method}

I participated in the physician training program of ASUISHI (to lead the im- 
provement of the quality of tomorrow's healthcare from January 2018 to June 2018, which was operated by the department of quality and safety management in the University of Nagoya and Quality Management at the department of the Toyota Motor Corp.). The aim of this ASUISHI program was the training of a manager on patient safety, infection control, and quality management. By attendance of 140 hours over 6 months of the ASUISHI program, I learned the method almost quality improvement by the industry total quality management [12] [13] [14] [15]. I tried to implement the quality improvement method for the infection control of our surgical ward. The methods of problem solving are performed in the 8 steps of 1) theme selection, 2) current situation, 3) setting a goal, 4) factor analysis, 5) measures planning, 6) measures execution, 7) effect confirmation, and 8) standardization and fixing of management.

\section{Results}

\subsection{Retrospective Analysis of the Patients during the MRSA Outbreak}

At the MRSA outbreak, ten MRSA patients were present in the same surgical ward ( 49 beds), and accounted for $20.4 \%$ of the total number of hospitalized patients (10/49); these patients were in the cardiovascular $(n=4)$, digestive $(n=3)$ and thoracic surgery $(n=3)$ units.

Table 1 and Table 2 show the breakdown of the 10 patients summarized by the similarity of drug susceptibility, same room during the same time period, nursing necessity, surgery and medical treatment, complications, detected site of MRSA, clinical course of MRSA, and outcomes. Regarding the same room during the same time period, three cases (cases 1,2 and 3) had a history of having entered the same hospital room A in the middle of April 2011, and another three cases (cases 4, 5 and 6) were found to have entered the same hospital room B in late May 2011. Five cases (cases 1, 4, 5, 6, and 8) showed similarities in drug susceptibility to MRSA, three of which (cases 4,5 and 6) had been admitted to the same room B at the same time, and the other 2 (cases 1 and 8) showed no similarity. Regarding the nursing necessity, seven cases (from case 1 to case 7) cannot be free to move on their own from the bed and they needed assistance, and the other three cases (cases 8, 9, and 10) could freely move from the hospital room without a need of assistance. Based on this, based on the history of the same room at the same time period, due to the similarity of drug susceptibility, the nursing necessity for assistance, etc., the occurrence of the MRSA outbreak was caused by the transmission within the same ward through the medical staff (between the doctor's groups and responsible nurses).

Figure 1 shows the situation of the 2-year term from April 2009 to May 2011 before the MRSA outbreak. The average amount of alcohol use for hand hygiene had showed the low level of 6 to $15 \mathrm{ml} /$ patient/day, values of which were extremely low and meant 2 to 5 times of hand sanitization (as a $3 \mathrm{ml}$ of alcohol amount is used for 1 time of hand hygiene by one push of the alcohol sanitizer 


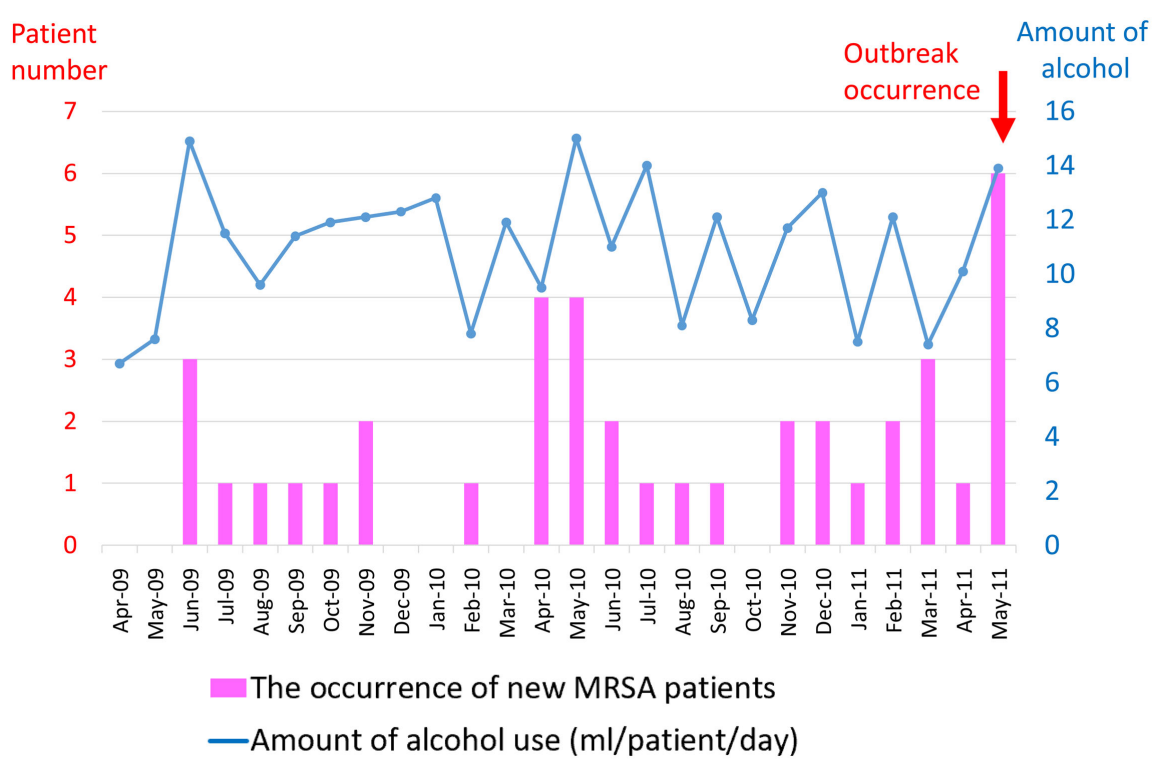

Figure 1. Averaged amount of alcohol use (ml/patient/day) and number of new MRSA patients before MRSA outbreak (April 2009 to May 2011).

bottle). In those days, the number of occurrences of new MRSA patients before the MRSA outbreak was usually detected from 1 and 6 patients per one month. New MRSA patients had been frequently detected in very many numbers in almost every month. Lastly, the MRSA outbreak resulted in the occurrence in May 2011.

Table 2 shows the summary of the short-term outcomes in those days with the ten MRSA outbreak patients. Regarding complications, the detected site of the MRSA, the clinical course of MRSA, and the outcomes, in two cases, the MRSA-infected wounds were covered with muscle flap and the clinical symptoms subsided. Regarding the clinical course of the ten MRSA outbreak patients, the outcome of MRSA resulted in it finally disappearing $(n=6)$, settled $(n=3)$, and patient died due to acute exacerbation of renal failure $(n=1)$.

\subsection{Short-Term Outcome during the Period before and after the Outbreak}

Figure 1 shows the results of the 2-year period before the MRSA outbreak from April 2009 to May 2011, which displays the outcome of the very low level performance of infection control, that is, the decreased amount of alcohol use for hand hygiene (ml/patient/day) and the high level of the occurrence of new MRSA patients. During this period, the amount of alcohol used per patient per day fluctuates in the range of 6 to $15 \mathrm{ml} /$ patient/day for about 1 year before the MRSA outbreak. Retrospectively, the level in those days was extremely low. In April and May 2011, during the two months before the occurrence of the outbreak, the monthly average value was very low and calculated as 10 and 14 $\mathrm{ml} /$ patient/day. Finally, the MRSA outbreak had occurred in our surgical ward in June 2011. In June 2011, the nosocomial center for infection control (CIC) 
intervened with emergent infection control in our surgical ward. We followed the emergent infection prevention measures for our hospitalized patients and we implemented the agreed items regarding dressing-exchange of the surgical site.

Figure 2 shows the dramatic change in the amount of alcohol use and the occurrence of new MRSA patients before and after the outbreak. After the alcohol hand disinfection was thoroughly implemented, the monthly average alcohol consumption per patient per day showed a dramatic increase. In June and July 2011, these months had shown a dramatic increase in the monthly alcohol use to an average of $106,71 \mathrm{ml} /$ patient/day respectively, thus it had been maintained in the range of 42 to $76 \mathrm{ml} /$ patient/day for one year. In June, July, and August 2011, in which we implemented a newly revised infection control method, no new MRSA patients had been observed for one month in June 2011. Although only 2 and 1 patients were detected in July and August 2011 respectively, however, the incidence of new MRSA patients underwent a drastic decline. It took three months from the start of strict infection control to stop the MRSA outbreak. Intervention and guidance from the center for infection control (CIC) about the outbreak had been carried out in the surgical ward. After that, the number of newly MRSA-detected cases was 0 for the 5 month period from September 2011 to January 2012.

In Figure 2, the number of new MRSA patients and the monthly average alcohol consumption were compared in a time series. Prior to the outbreak, the alcohol consumption for hand hygiene was very low, in contrast, it was found that the number of new MRSA outbreaks was constantly occurring every month. It was found that after the outbreak, the nosocomial center for infection control (CIC) intervened in our surgical ward, our team approach for infection control

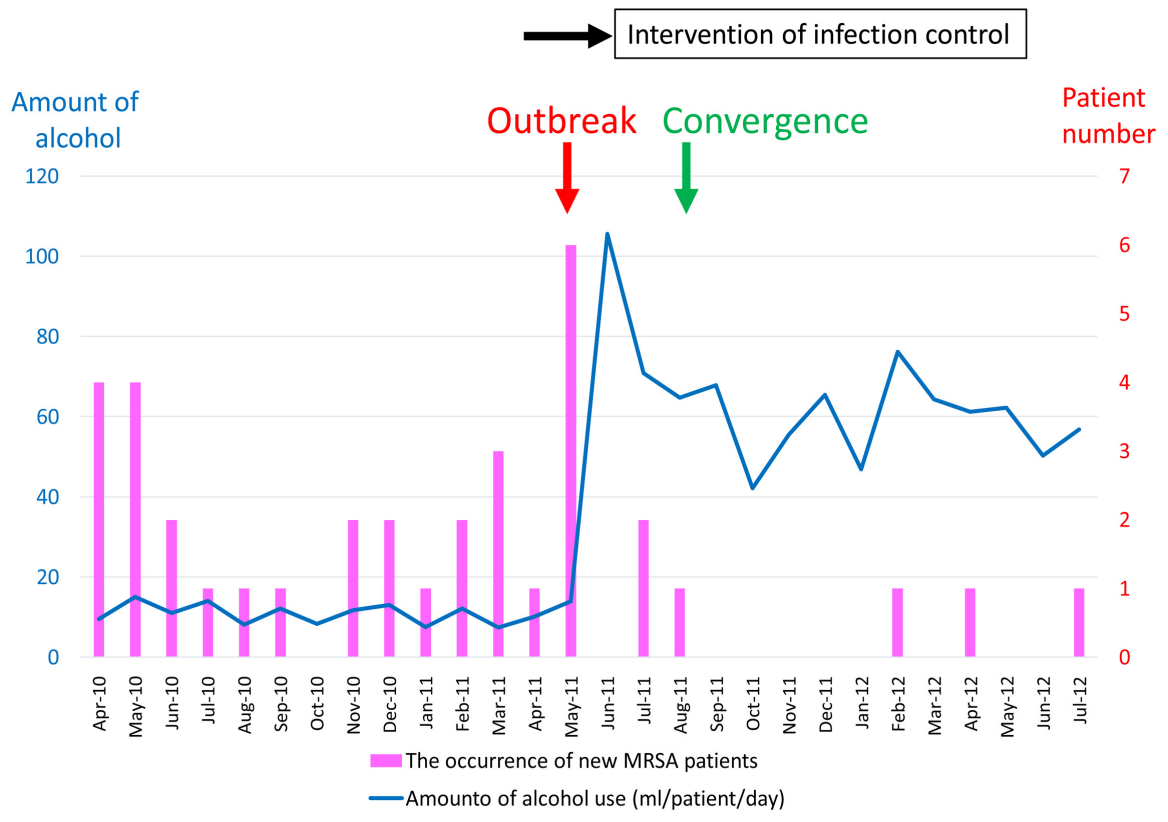

Figure 2. Short-term outcome of changing the amount alcohol use for hand hygiene (ml/patient/day) and occurrence of new MRSA patients. 
dramatically increased the alcohol use for hand hygiene and consequently reduced the number of new MRSA patients. Figure 2 shows that an inverse correlation was suggested between the incidence of new MRSA patients and alcohol consumption. It has been empirically proved that a thorough manual alcohol disinfection is effective in preventing MRSA transmission.

\subsection{Long-Term Outcome after the Convergence of Outbreak}

Figure 3 shows the long-term transition, that is, 6 years of occurrences of new MRSA patients and the monthly average alcohol use ( $\mathrm{ml} /$ patient/day) after the outbreak. However, the novel experience of the outbreak has been diluted, thus the thorough hand hygiene and the manual compliance of infection control have become disregarded. In July 2013, the monthly average alcohol use for hand hygiene had gradually decreased and the level did not exceed the level of 60 $\mathrm{ml} /$ patient/day.

In December 2014, 3 years after the outbreak, the monthly average alcohol use had decreased to a level of $40 \mathrm{ml} /$ patient/day. New MRSA patients have occasionally occurred and the risk of a recurrence of the outbreak had become high. As Figure 3 shows, although there had not been any new occurrences of a MRSA outbreak, however, the new MRSA patients have not been cured. We recognized that it was difficult to maintain a strict infection control with a sense of tension and it became a challenge.

\subsection{Results of the Intervention of Industry Problem-Solving Methods for the Eradication of New MRSA Patient Occurrences}

To eradicate any new occurrence of MRSA patients, we newly implemented an

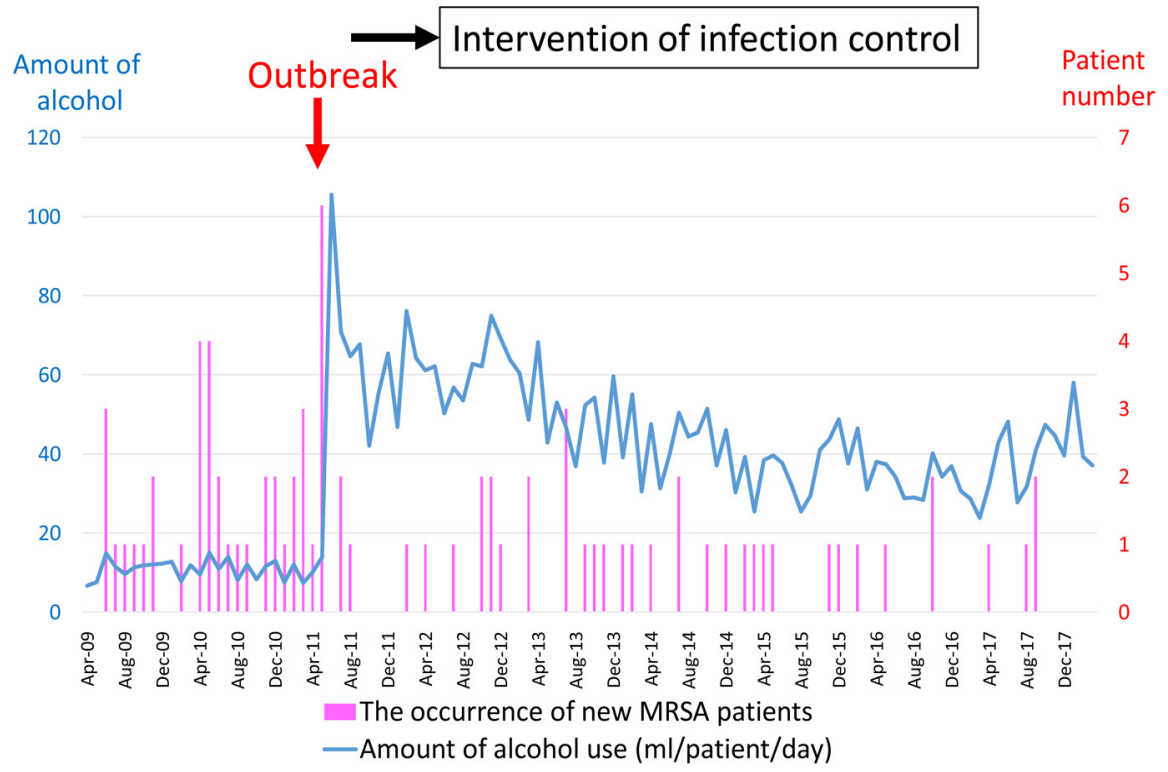

Figure 3. Long-term outcome of alcohol use for hand hygiene ( $\mathrm{ml} /$ patient/day) and occurrence of new MRSA patients. 
industry total quality management and problem-solving method for infection control. The methods of problem-solving were performed in 8 steps; step-1: theme selection, step-2: current situation, step-3: setting a goal, step-4: factor analysis, step-5: measures planning, step-6: execution measures, step-7: effect confirmation, and step-8: standardization and fixing of management.

As step-1, "theme selection" was summarized that: 1) the MRSA outbreak occurred 6 years ago ( 6 new patients, 10 patients/49 patient beds, 20.4\%); 2) from the analysis of the detection status of MRSA (same room in the same period, similar drug susceptibility and nursing necessity), it should be possible to cause the propagation by the medical staff in the same ward (physician intergroup and nurse in charge); 3) planning of the infection prevention measures and reviewing of hand hygiene method, and complying with the instructions of dressing exchange of surgical wounds; 4 ) the experience of MRSA outbreak has been forgotten for 6 years; 5) disregard of the manual compliance and the infection prevention measures; 6) decreasing of the monthly averaged alcohol use amount (ml/patient/day) and occasional occurrence of new MRSA patients; 7) by surgical ward reorganization from April 2018, the occurrence risk of new MRSA patients increased, thus the need of urgent measures for eradication of the MRSA occurrence.

As for step-2, the "current situation and understanding" was summarized that loss of the MRSA outbreak experience; countermeasures and non-compliance of manuals; decreased number of hand hygiene treatments; no review of manual regarding infection control (the last version was February, 2012); delay of early-detection of a MRSA patient; insufficient cases of contact infection prevention is random; shortage of private rooms for multiple infected MRSA patients; inappropriate uses of antibiotics over postoperative 48 hours; no education and no drill for newcomers and medical staff; no leader of infection control; decreases in behavior of infection prevention and crisis consciousness; and increasing the risk of infection by reorganization of surgical ward in April, 2018.

Figure 4 shows the current situation and the trend in the amount alcohol use and number of new MRSA patients in the surgical ward (April 2012 to March 2018). From April 2015, the amount of alcohol use showed a low level between 25 and $45 \mathrm{ml} /$ patient/day. Although there was no occurrence of a MRSA outbreak, however, new MRSA patients occasionally occurred. The indicator of the value " $45 \mathrm{ml} /$ patient/day" means about 15 hand hygiene times ( 1 time of hygiene is about equal to $3 \mathrm{ml}$ of alcohol use). After the MRSA outbreak, the number of hand hygiene treatments gradually decreased. New MRSA patients then occasionally occurred. We intended the goal to be zero occurrence of new MRSA patients from April 2018.

As step-3, "setting a goal" was to stop any new occurrence of MRSA patients in the surgical ward, thus we set a goal. 1) from April 2018, we intended to increase the averaged amount of alcohol use up to $40 \mathrm{ml} /$ patient/day (the averaged hand hygiene times up to 14 times/patient/day). In June 2018, we increased the 


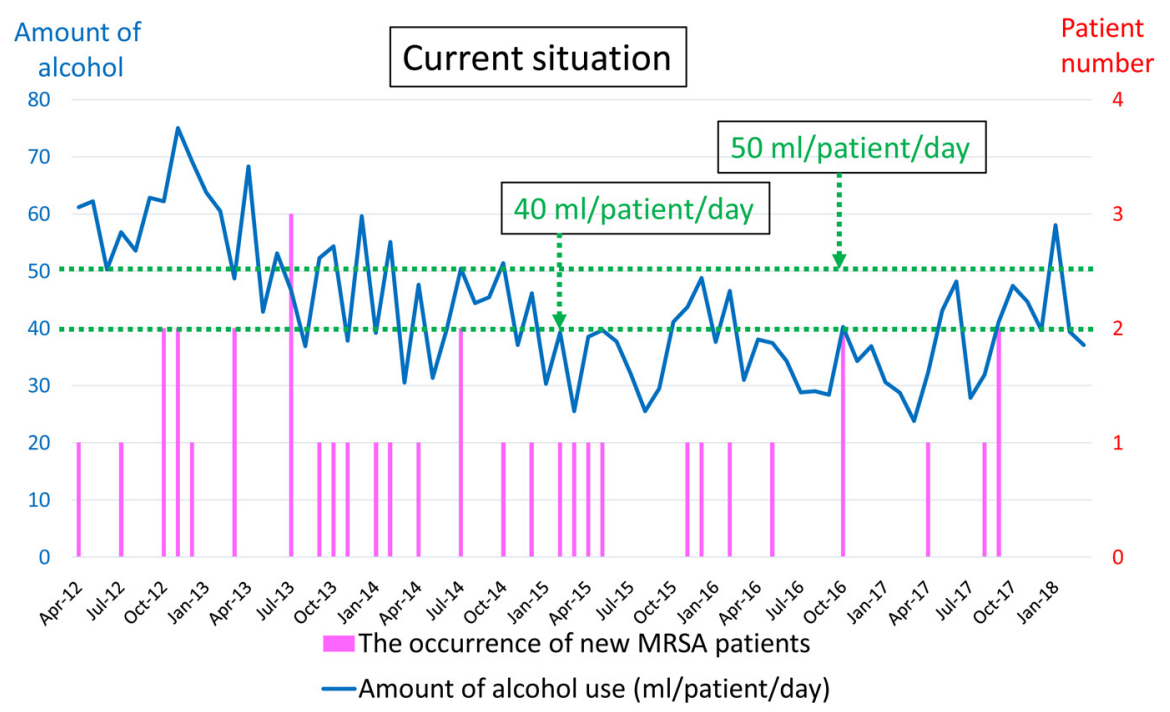

Figure 4. Long-term transition of MRSA patient occurrence after intervention of infection control. Current situation.

averaged amount of alcohol use to $50 \mathrm{ml}$ (17 times/patient/day). 2) from April 2018, we intended to maintain zero new occurrences of MRSA patients in the surgical ward.

As step-4, a "factor analysis" was performed on the root cause of the occurrence of new MRSA patients. Figure 5 shows a cause and effect diagram with five branches that are the patient, medical staff, method (skills/tools), team/organization, and task/environment. We focused on the true factors; 1) number of everyday hand hygiene is few, and the amount of alcohol use is less, 2) there was no reviewing of procedures and check sheet; 3 ) delay in determining MRSA-detected patient, and 4) no leader of infection control, and no education and no training of new staff.

As step-5, "measures planning, measures execution, and effective confirmation" are summarized: 1) daily averaged amount of alcohol use, and monitoring of times of hand hygiene. From April 2018, the averaged amount of alcohol use for hand hygiene increased to more than $40 \mathrm{ml}$ ( $\mathrm{ml} /$ patient/day). The averaged times of hand hygiene increased to more than 14 (times/patient/day); 2) standard procedures of infection control, and reviewing of check sheet. From April 2018, every morning, updating of check sheet regarding the patient's wound situation (open, infection, toileting, and drain tube). Implementation of a new standard regarding the agreement on the procedure of wound dressing; 3) early detection of MRSA-patient and setting of special management of MRSA-infected patients. From April 2018, checking of MRSA-patient at admission. Early culture inspection for the suspected patient with MRSA infection. Understanding of the location of MRSA positively-detected patient; 4) nomination of leader for infection control, and education and training of new comers and medical staff. From April 2018, a leader for infection control educates and supervises the standard procedure and checklist to the medical staff. Holding of regulatory study 


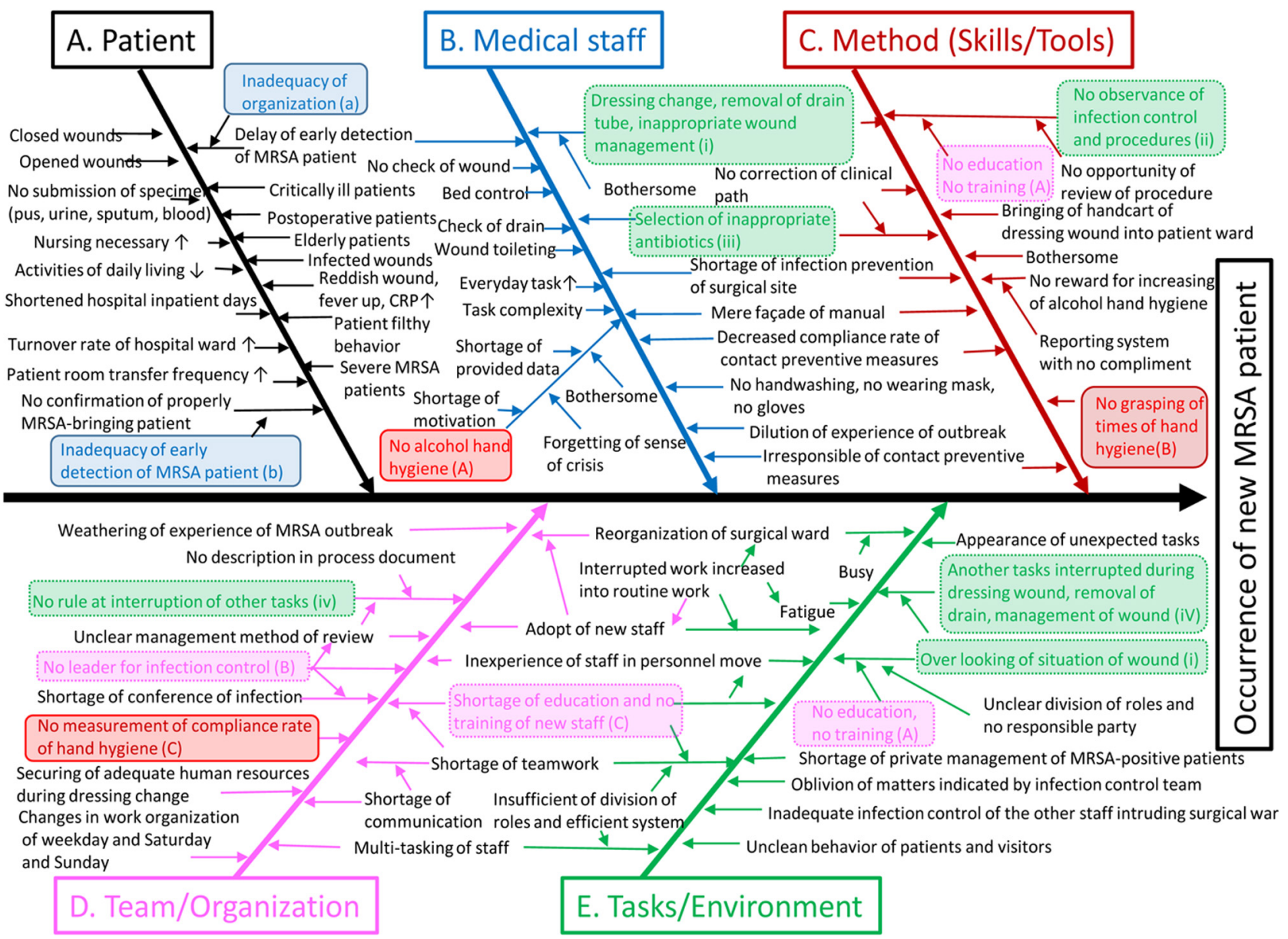

Figure 5. Factor analysis.

meetings and confirmation of level of understanding.

As step-6, "standardization, definition of management and comprehensive evaluation" are 1) sustaining of a new standardization for the manual of standard precaution and contact infection measures, and arrangements of wound dressing; 2) regarding a new standardization of infection control, we intend to have regular study workshops of teaching and training; 3) comprehensive evaluation: from April, 2018, the risk of MRSA occurrence increased. Although one new MRSA patient was observed in May 2018, however, infection prevention measures have been continually performed. Figure 6 shows the A3 problem solving regarding the agglomeration of the Kaizen approach.

\subsection{Outcome of the Implementation of the Quality Improvement with Problem-Solving Method}

As step-7, Figure 7 displays the "effect confirmation", which shows the effectiveness of this industry problem-solving approach to infection control. As a lower limit of the hand hygiene, we set the goal of over the level of 40 $\mathrm{ml} /$ patient/day (14 times of hand hygiene), the intervention was started in April 2018, but a new MRSA patient was detected in May 2018. The time overlaps 


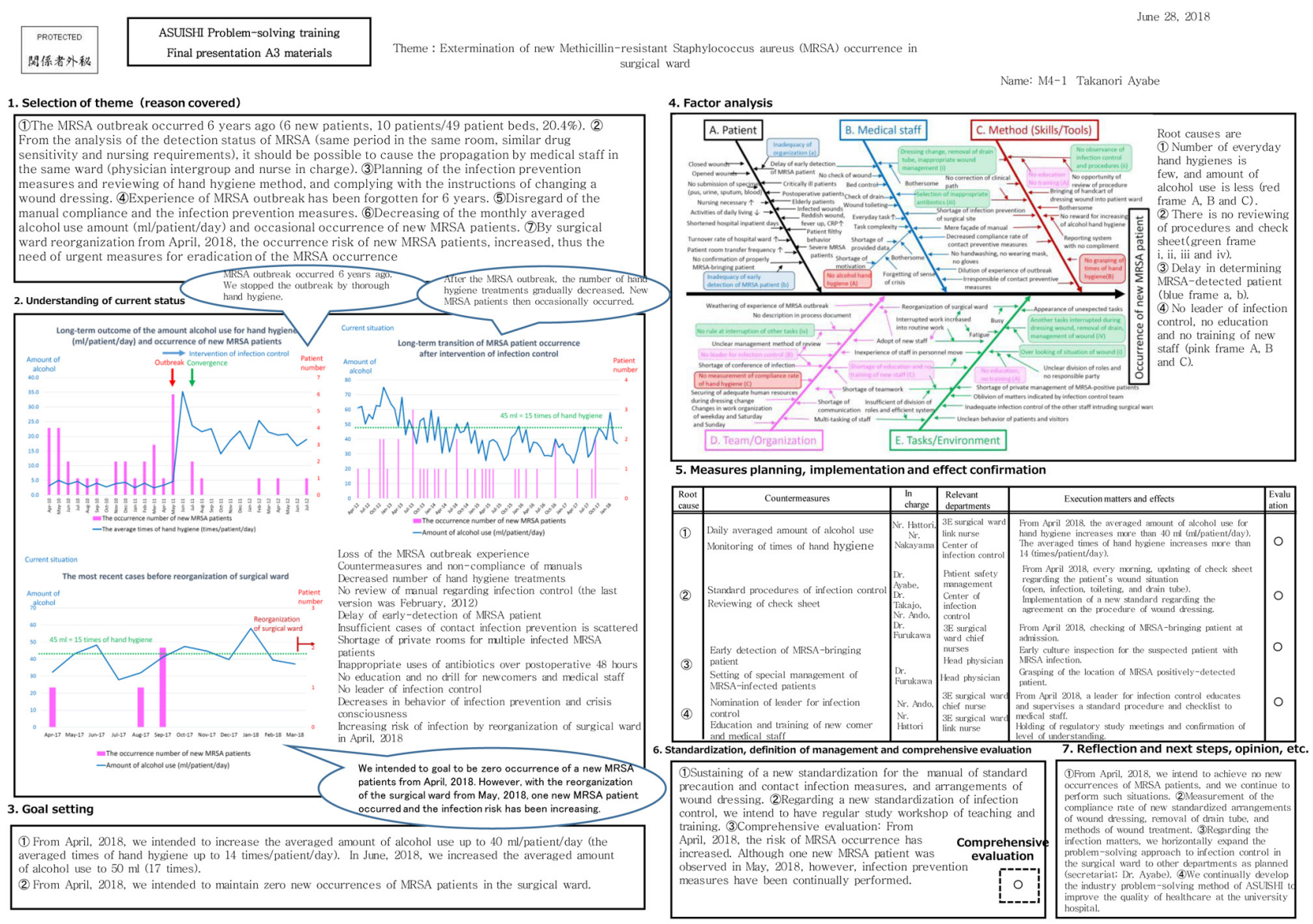

Figure 6. A3 sheet.

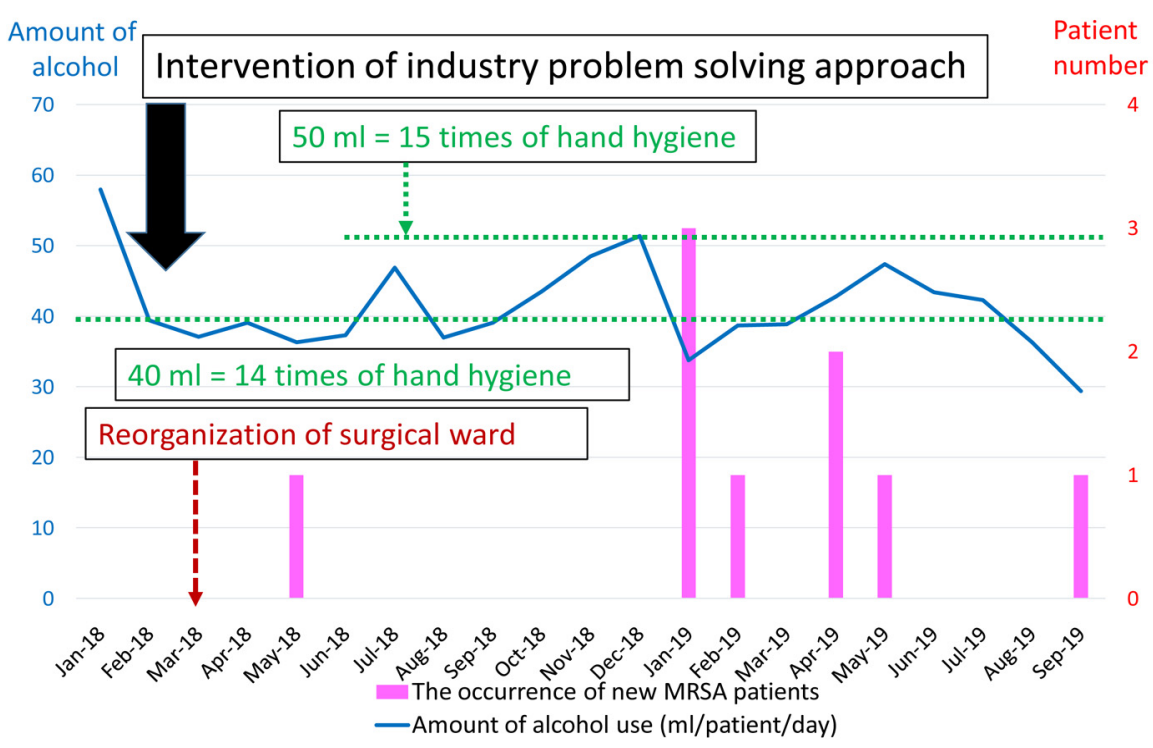

Figure 7. Outcomes of intervention of industry problem solving approach (between January, 2018 and October, 2019).

when the surgical ward had been reorganized in April 2018. Once the amount of alcohol use for hand hygiene was reduced below the level of $30 \mathrm{ml} /$ patient/day, 
new MRSA patients occurred, however, the use of alcohol reached the level of 45 $\mathrm{ml} /$ patient/day, the amount of usage in May 2019. In June 2018, we increased the lower limit of the amount of alcohol use to be over the level of $50 \mathrm{ml} /$ patient/day (15 hand hygiene times). There has been no detection of new MRSA patients for 7 months between June 2018 and December 2018. However, three new MRSA patient was detected in January 2019, when the amount of the alcohol use for hand hygiene had decreased to less than the level of $40 \mathrm{ml} /$ patient/day. There displayed an inverse correlation between the increased number of new MRSA patients and the decreased amount of alcohol use for hand hygiene.

As step-8, "standardization and fixing of management" are described as follows: 1) from April 2018, we intended to achieve no new occurrences of MRSA patients, and we continue to perform such a goal, 2) measurement of the compliance rate of new standardized arrangements of wound dressing, removal of drain tube, and methods of wound treatment, 3) regarding the infection matters, we expanded the problem-solving approach to infection control in the surgical ward to other departments as planned (secretariat; Dr. Ayabe), and 4) we continually developed the industry problem-solving method of ASUISHI to improve the quality of healthcare at the university hospital.

\section{Discussion}

Health-care-associated infections represent a leading preventable adverse event in patients [16]. Methicillin-resistant Staphylococcus aureus is a major cause of hospital-acquired infections in many countries around the world [17] [18]. In the last decade, numerous reviews and consensus statements have endorsed policies to control the spread of nosocomial MRSA infections [19] [20] [21].

Hand hygiene is widely considered the key to prevent such infections and cross-transmission of multidrug-resistant organisms [1] [22]. WHO recommends a multimodal approach to hand hygiene promotion that includes provision of alcohol-based hand rub at the point of care, education of healthcare workers, audit and performance feedback of hand hygiene behavior, reminders in the workplace, and institutional safety culture. Existing evidence supports the effectiveness of multimodal hand hygiene promotion [1] [16] [22]-[26]. However, sustaining success remains challenging. Regarding the patient's hand washing, it is just as important as hospital workers' hand hygiene. The increased hand hygiene compliance by patients can influence the infection rates in an adult cardiothoracic step-down unit [27]. The decreased infection rates and increased compliance with hand hygiene among the patients may be attributed to the implementation of patient education and the increased accessibility and use of hand sanitizers [27].

Pittet et al. [8] reported that hand hygiene adherence was associated with lower MRSA colonization rates [8]. Bischoff et al. [28] also observed that improved accessibility to alcohol-based hand sanitizers enhanced adherence to hand hygiene. In our surgical ward in those days (Figure 1), the daily averaged 
amount of alcohol consumption per patient was calculated to be the low level of 8 to $14 \mathrm{ml} /$ patient/day. As one complete pushing volume from the alcohol hand sanitizer supply bottle can be measured by about $3 \mathrm{ml}$, this amount can be counted as 1 hand hygiene time. Retrospectively, we considered the status of hand hygiene before the MRSA outbreak in past days. If the hand disinfection is performed twice before and after wound treatment for one patient care, in the calculation, at least $6 \mathrm{ml}$ of alcohol (two pushes of alcohol supply bottle) is used for one nursing care or doctor's round for 1 patient. At that time, the levels of 8 to $14 \mathrm{ml} /$ patient/day, which means that only 2 or 3 hand disinfection times were performed for one patient care for a day, was a situation that meant incredibly very low levels of hand hygiene in those days. If 10 times the patient care and wound treatment per one patient for a day were performed, the amount of alcohol use would be estimated to be at least more than the level of $60 \mathrm{ml} /$ patient/day. Based on the data obtained from the surgical ward during the MRSA outbreak (Figure 2), it was observed that if the used alcohol amount was not below the lowest level of $40 \mathrm{ml} /$ patient/day, this level showed that there was decrease in new occurrences of MRSA patients. We have continued to monitor the daily alcohol consumption in the surgical ward in order not to decrease the level of 60 $\mathrm{ml} /$ patient/day (Figure 2). This monitoring system serves as a convenient tool to visualize the hand hygiene status. For example, if the alcohol consumption decreased below the level of $40 \mathrm{ml} /$ patient/day (equivalent to 6 to 7 hand hygienetimes), we can alert to the multi-professional staff the decreased status of the disinfection level and we can call for more implementation of hand hygiene in order to increase to more than the level of $60 \mathrm{ml} /$ patient/day (Figure 2). Since then, the raised awareness for infection control has been maintained in our surgical ward.

Figure 5 and Figure 6 show the A3 sheet summary by the problem-solving method, that is the Kaizen approach for the eradication of new MRSA occurrences in the surgical ward, which is what we complied for the process of industry problem-solving with the team staff. In is important for the team members to understand and share the 8 steps of the problem-solving approach, that is to select the theme, to investigate current situation, to set a goal, to analyze factors, to measure planning, to measure execution, to confirm effects, and standardize and to fix the management. The A3 sheet (Figure 6) included these 8 steps and visualized the total quality management in surgical ward, which displays the responsible person in rule.

The limitation of the present study is the outcome in the small surgical ward section in a single university hospital, which is based on the dynamic implementation of changing infection control activity by feedback of the daily monitoring of the level of hand hygiene, and which was retrospectively analyzed. The long-term maintaining of compliance of hand hygiene is actually very difficult. Although performing alcohol hand hygiene is understood to be very good evidence for infection control, however, the implementation and the sustainable 
practice are very difficult to perform. There are some gaps between the evidence and the practice in the workplace.

As our future direction, we will expand the Kaizen approach across a section of our hospital. Although the quality improvement, such as industry problem-solving [12] [13] and the Lean Six sigma tool [15], is based on the Japanese Kaizen approach [14], these implementations and the continuity are very useful. Kaizen is a Japanese word that means "change for the better," as popularized by Masaaki Imai [14]. In order to expand the efforts for infection prevention, not only an individual effort but also a multi-professional team effort are cross-organizationally expected to form a culture of infection control, that is, as an organization culture, both top-down instructions and a bottom-up approach are important to implement infection control measures. The organization culture is defined as the assumptions, values, and norms shared among colleagues. These beliefs are often taken for granted, but have the ability to influence an individual's thinking and behavior [29]. The organization culture can impact (positively or negatively) the organizational success and an organization's ability to implement change. The organization culture has recently gained recognition in the health care setting and is an element to consider when implementing an infection control program and prevention initiatives [30] [31] [32] [33]. Findings from several research studies focusing on infection control have confirmed the association between a patient's outcome and various organization culture factors, including leadership, teamwork, staff satisfaction, staff morale, and workload [1] [34]. More specifically, having weak/poor levels of these factors (i.e., poor organization culture) are considered risks for infection control problems and adverse patient outcomes [35] [36]. Additional research in the intensive care unit setting has shown that effective leadership and communication can lead to better team cohesiveness and overall performance [37] [38].

Lastly, by the retrospective analysis regarding the short-term outcome of infection control during the MRSA outbreak and the convergence, the number of new MRSA patients was negatively related to the volume of everyday alcohol consumption. We succeeded in ending the MRSA outbreak by a team approach to controlling infection for 3 months. Hand hygiene with alcohol use should be effective in preventing MRSA transmission. The level of alcohol consumption for hand washing has been maintained at 40 to $80 \mathrm{ml} /$ patient/day, and almost no MRSA patients had been observed for one year. By the evaluation regarding the long-term outcome of a 7 years' infection control after convergence of the outbreak, the amount of alcohol use has gradually decreased and MRSA patients have been occasionally observed, thus these facts should display the difficulty in maintaining a strict infection control. Regarding compliance of hand hygiene and occurrence of MRSA patients, bridging the gap between ideal and reality and how to reconcile is difficult but very important. It is effective to start a Kaizen approach with an industry problem-solving method for the eradication of a new occurrence of MRSA patients, which efforts can be visualized by creating 
the process in an A3 sheet (Figure 6). It is useful for a multidisciplinary approach to perform the improvement of the quality and the activity of infection control by teamwork.

\section{Conclusion}

Based on the more than 8-year long-term outcome of infection control by measuring the daily amount of alcohol use for hand hygiene and the directly-connected prevention measures for new MRSA patients, these can reconcile the theory of infection control and the implementation of everyday practical hand hygiene. By creating and performing a Kaizen approach with the problem-solving method, which can realize 7 months of zero new occurrences of MRSA and will contribute to the multi-professional team to visualize the process of quality improvement for infection control. There was an inverse correlation between the decreased amount of alcohol use for hand hygiene and the increased number of new MRSA patients.

\section{Acknowledgements}

We appreciate the members of the ASUISHI program (Yoshimasa Nagao, Ayuko Yasuda, and Tetsuya Yagi, who are instructors of the ASUISHI project promotion office in the Nagoya University Hospital, and Kazuo Umemoto, the instructor from the Central Japan Quality Control Association). This study was supported by a Grant-in-Aid for Clinical Research from the Miyazaki University Hospital.

\section{Conflicts of Interest}

The authors declare no conflicts of interest regarding the publication of this paper.

\section{References}

[1] World Health Organization (2009) WHO Guidelines on Hand Hygiene in Healthcare: First Global Patient Safety Challenge Clean Care Is Safer Care. World Health Organization Press, Geneva, Switzerland.

[2] Centers for Disease Control and Prevention (2014) Hand Hygiene Basics. http://www.cdc.gov/handhygiene/Basic.html

[3] Centers for Disease Control and Prevention (2016) Hand Hygiene in Healthcare Settings. http://www.cdc.gov/handhygiene/

[4] Grundmann, H., Aires-de-Sousa, M., Boyce, J. and Tiemersma, E. (2006) Emergence and Resurgence of Meticillin-Resistant Staphylococcus aureus as a Public-Health Threat. The Lancet, 368, 874-885. https://doi.org/10.1016/S0140-6736(06)68853-3

[5] McDanel, J.S., Ward, M.A., Leder, L., Schweizer, M.L., Dawson, J.D., Diekema, D.J., Smith, T.C., Chrischilles, E.A., Perencevich, E.N. and Herwaldt, L.A. (2014) Methicillin-Resistant Staphylococcus aureus Prevention Practices in Hospitals throughout a Rural State. American Journal of Infection Control, 42, 868-873. https://doi.org/10.1016/j.ajic.2014.05.004 
[6] Boyce, J.M. (2001) MRSA Patients: Proven Methods to Treat Colonization and Infection. Journal of Hospital Infection, 48, S9-S14. https://doi.org/10.1016/S0195-6701(01)90005-2

[7] Reybrouck, G. (1986) Handwashing and Hand Disinfection. J Hosp Infect, 8, 5-23. https://doi.org/10.1016/0195-6701(86)90100-3

[8] Pittet, D., Hugonnet, S., Harbarth, S., Mourouga, P., Sauvan, V., Touveneau, S. and Perneger, T.V. (2000) Effectiveness of a Hospital-Wide Programme to Improve Compliance with Hand Hygiene. Infection Control Programme. The Lancet, 356, 1307-1312. https://doi.org/10.1016/S0140-6736(00)02814-2

[9] Whitby, R.M. and McLaws, M.L. (2005) Efficacy of an Alcohol/Chlorhexidine Hand Hygiene Program in a Hospital with High Rates of Nosocomial Methicillin-Resistant Staphylococcus aureus (MRSA) Infection. Medical Journal of Australia, 183, 509-519. (Medical Journal of Australia, 2006, 184, 253-254; author reply 254)

[10] Albrich, W.C. and Harbarth, S. (2008) Health-Care Workers: Source, Vector, or Victim of MRSA? The Lancet Infectious Diseases, 8, 289-301. https://doi.org/10.1016/S1473-3099(08)70097-5

[11] Davis, C.R. (2010) Infection-Free Surgery: How to Improve Hand-Hygiene Compliance and Eradicate Methicillin-Resistant Staphylococcus aureus from Surgical Wards. Annals of the Royal College of Surgeons of England, 92, 316-319. https://doi.org/10.1308/003588410X12628812459931

[12] Jeffrey, L., (2003) The Toyota Way: 14 Management Principles from the World's Greatest Manufacturer. McGraw-Hill Education, New York.

[13] Katsuya, H. (2000) The QC Problem Solving Approach. Solving Workplace Problems the Japanese Way. JUSE Press, Tokyo, Japan.

[14] Masaaki, I. (2012) Gemba Kaizen: A Commonsense Approach to a Continuous Improvement Strategy. 2nd Edition, McGraw-Hill Education, New York.

[15] Jay, A. (2016) Lean Six Sigma for Hospitals: Improving Patient Safety, Patient Flow and the Bottom Line. 2nd Edition, McGraw-Hill Education, New York.

[16] Zingg, W., Holmes, A., Dettenkofer, M., Goetting, T., Secci, F., Clack, L., Allegranzi, B., Magiorakos, A.P. and Pittet, D. (2015) Systematic Review and Evidence-Based Guidance on Organization of Hospital Infection Control Programmes (SIGHT) Study Group. Hospital Organisation, Management, and Structure for Prevention of Health-Care-Associated Infection: A Systematic Review and Expert Consensus. The Lancet Infectious Diseases, 15,212-224. https://doi.org/10.1016/S1473-3099(14)70854-0

[17] Klevens, R.M., Morrison, M.A., Nadle, J., Petit, S., Gershman, K., Ray, S., Harrison, L.H., Lynfield, R., Dumyati, G., Townes, J.M., Craig, A.S., Zell, E.R., Fosheim, G.E., McDougal, L.K., Carey, R.B. and Fridkin, S.K. (2007) Active Bacterial Core surveillance (ABCs) MRSA Investigators. Invasive Methicillin-Resistant Staphylococcus aureus Infections in the United States. JAMA, 298, 1763-1771.

https://doi.org/10.1001/jama.298.15.1763

[18] Boucher, H.W. and Corey, G.R. (2008) Epidemiology of Methicillin-Resistant Staphylococcus aureus. Clinical Infectious Diseases, 46, S344-S3449. https://doi.org/10.1086/533590

[19] Siegel, J.D., Rhinehart, E., Jackson, M. and Chiarello, L. (2007) Healthcare Infection Control Practices Advisory Committee. Management of Multidrug-Resistant Organisms in Health Care Settings, 2006. American Journal of Infection Control, 35, S165-S193. https://doi.org/10.1016/j.ajic.2007.10.006

[20] Gould, F.K., Brindle, R., Chadwick, P.R., Fraise, A.P., Hill, S., Nathwani, D., Ridg- 
way, G.L., Spry, M.J. and Warren, R.E., MRSA Working Party of the British Society for Antimicrobial Chemotherapy (2009) Guidelines (2008) for the Prophylaxis and Treatment of Methicillin-Resistant Staphylococcus aureus (MRSA) Infections in the United Kingdom. Journal of Antimicrobial Chemotherapy, 63, 849-861. https://doi.org/10.1093/jac/dkp065

[21] Zoabi, M., Keness, Y., Titler, N. and Bisharat, N. (2011) Compliance of Hospital Staff with Guidelines for the Active Surveillance of Methicillin-Resistant Staphylococcus aureus (MRSA) and Its Impact on Rates of Nosocomial MRSA Bacteremia. The Israel Medical Association Journal, 13, 740-744.

[22] Ellingson, K., Haas, J.P., Aiello, A.E., Kusek, L., Maragakis, L.L., Olmsted, R.N., Perencevich, E., Polgreen, P.M., Schweizer, M.L., Trexler, P., VanAmringe, M. and Yokoe, D.S. (2014) Society for Healthcare Epidemiology of America (SHEA). Strategies to Prevent Healthcare-Associated Infections through Hand Hygiene. Infection Control \& Hospital Epidemiology, 35, 937-960. https://doi.org/10.1086/651677

[23] Schweizer, M.L., Reisinger, H.S., Ohl, M., Formanek, M.B., Blevins, A., Ward, M.A. and Perencevich, E.N. (2014) Searching for an Optimal Hand Hygiene Bundle: A Meta-Analysis. Clinical Infectious Diseases, 58, 248-259. https://doi.org/10.1093/cid/cit670

[24] Allegranzi, B., Gayet-Ageron, A., Damani, N., Bengaly, L., McLaws, M.L., Moro, M. L., Memish, Z., Urroz, O., Richet, H., Storr, J., Donaldson, L. and Pittet, D. (2013) Global Implementation of WHO's Multimodal Strategy for Improvement of Hand Hygiene: A Quasi-Experimental Study. The Lancet Infectious Diseases, 13, 843-851. https://doi.org/10.1016/S1473-3099(13)70163-4

[25] Rosenthal, V.D., Pawar, M., Leblebicioglu, H., Navoa-Ng, J.A., Villamil-Gómez, W., Armas-Ruiz, A., Cuéllar, L.E., Medeiros, E.A., Mitrev, Z., Gikas, A., Yang, Y., Ahmed, A., Kanj, S.S., Dueñas, L., Gurskis, V., Mapp, T., Guanche-Garcell, H., Fernández-Hidalgo, R. and Kübler, A. (2013) Impact of the International Nosocomial Infection Control Consortium (INICC) Multidimensional Hand Hygiene Approach over 13 Years in 51 Cities of 19 Limited-Resource Countries from Latin America, Asia, the Middle East, and Europe. Infection Control \& Hospital Epidemiology, 34, 415-423. https://doi.org/10.1086/669860

[26] Luangasanatip, N., Hongsuwan, M., Limmathurotsakul, D., Lubell, Y., Lee, A.S., Harbarth, S., Day, N.P., Graves, N. and Cooper, B.S. (2015) Comparative Efficacy of Interventions to Promote Hand Hygiene in Hospital: Systematic Review and Network Meta-Analysis. BMJ, 351, h3728. https://doi.org/10.1136/bmj.h3728

[27] Haverstick, S., Goodrich, C., Freeman, R., James, S, Kullar, R. and Ahrens, M. (2017) Patients' Hand Washing and Reducing Hospital-Acquired Infection. Critical Care Nurse, 37, e1-e8. https://doi.org/10.4037/ccn2017694

[28] Bischoff, W.E., Reynolds, T.M., Sessler, C.N., Edmond, M.B. and Wenzel, R.P. (2000) Handwashing Compliance by Health Care Workers: The Impact of Introducing an Accessible, Alcohol-Based Hand Antiseptic. Archives of Internal Medicine, 160, 1017-1021. https://doi.org/10.1001/archinte.160.7.1017

[29] Davies, H.T., Nutley, S.M. and Mannion, R. (2000) Organisational Culture and Quality of Health Care. BMJ Quality \& Safety, 9, 111-119.

https://doi.org/10.1136/qhc.9.2.111

[30] Pronovost, P. (2008) Interventions to Decrease Catheter-Related Bloodstream Infections in the ICU: The Keystone Intensive Care Unit Project. American Journal of Infection Control, 36, S171.e1-5. https://doi.org/10.1016/j.ajic.2008.10.008

[31] Pronovost, P.J., Berenholtz, S.M., Goeschel, C., Thom, I., Watson, S.R., Holzmuel- 
ler, C.G., Lyon, J.S., Lubomski, L.H., Thompson, D.A., Needham, D., Hyzy, R., Welsh, R., Roth, G., Bander, J., Morlock, L. and Sexton, J.B. (2008) Improving Patient Safety in Intensive Care Units in Michigan. Journal of Critical Care, 23, 207-221. https://doi.org/10.1016/j.jcrc.2007.09.002

[32] Pronovost, P., Needham. D., Berenholtz, S., Sinopoli, D., Chu, H., Cosgrove, S., Sexton, B., Hyzy, R., Welsh, R., Roth, G., Bander, J., Kepros, J. and Goeschel, C. (2006) An Intervention to Decrease Catheter-Related Bloodstream Infections in the ICU. The New England Journal of Medicine, 355, 2725-2732. https://doi.org/10.1056/NEJMoa061115

[33] Alexander, J.A., Weiner, B.J., Shortell, S.M., Baker, L.C. and Becker, M.P. (2006) The Role of Organizational Infrastructure in Implementation of Hospitals' Quality Improvement. Hospital Topics, 84, 11-20. https://doi.org/10.3200/HTPS.84.1.11-21

[34] Griffiths, P., Renz, A., Hughes, J. and Rafferty, A.M. (2009) Impact of Organisation and Management Factors on Infection Control in Hospitals: A Scoping Review. Journal of Hospital Infection, 73, 1-14. https://doi.org/10.1016/j.jhin.2009.05.003

[35] Scott, T., Mannion, R., Davies, H.T. and Marshall, M.N. (2003) Implementing Culture Change in Health Care: Theory and Practice. International Journal for Quality in Health Care, 15, 111-118. https://doi.org/10.1093/intqhc/mzg021

[36] Jain, M., Miller, L., Belt, D., King, D. and Berwick, D.M. (2006) Decline in ICU Adverse Events, Nosocomial Infections and Cost through a Quality Improvement Initiative Focusing on Teamwork and Culture Change. Quality and Safety in Health Care, 15, 235-239. https://doi.org/10.1136/qshc.2005.016576

[37] Ohman-Strickland, P.A., John Orzano, A., Nutting, P.A., Perry Dickinson, W., Scott-Cawiezell, J., Hahn, K., Gibel, M. and Crabtree, B.F. (2007) Measuring Organizational Attributes of Primary Care Practices: Development of a New Instrument. Health Services Research, 42, 1257-1273. https://doi.org/10.1111/j.1475-6773.2006.00644.x

[38] Wolf, R., Lewis, D., Cochran, R. and Richards, C. (2008) Nursing Staff Perceptions of Methicillin-Resistant Staphylococcus aureus and Infection Control in a Long-Term Care Facility. Journal of the American Medical Directors Association, 9, 342-346. https://doi.org/10.1016/j.jamda.2008.02.003 